\title{
TEMPERATURE MEASUREMENTS IN NON-EQUILIBRIUM "FERROELECTRIC" PLASMA
}

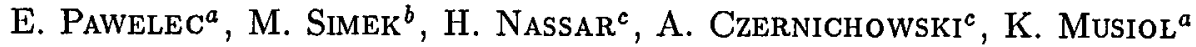 \\ AND L. DitTRICHOVA ${ }^{d}$ \\ ${ }^{a}$ Institute of Physics, Jagiellonian University, Reymonta 4, 30-059 Kraków, Poland \\ bInstitute of Plasma Physics, Prague, Czech Republic \\ ${ }^{c}$ GREMI, University of Orleans, 45067 Orleans cedex 2, France \\ ${ }^{d}$ Technical University, Brno, Czech Republic
}

(Received December 8, 1994; revised version September 28, 1995)

\begin{abstract}
Using spectroscopic measurements vibrational and rotational temperatures were determined in the "ferroelectric" plasma source for different gas mixtures. It was shown that in this time-periodical, atmospheric pressure non-equilibrium discharge, both plasma temperatures differ strongly, and that the vibrational temperature is much higher $(\approx 3 \mathrm{kK})$ than the rotational one $(<1 \mathrm{kK})$.
\end{abstract}

PACS numbers: $33.10 . \mathrm{Jz}, 52.70 . \mathrm{Kz}, 52.25 . \mathrm{Kn}$

\section{Introduction}

We have put attention to the so-called ferroelectric plasma, the periodic electrical discharge, which has been used for several applications in chemistry and spectroscopic measurements [1]. This low power (about few watts) discharge, originating from repolarization of an ferroelectric plate in electric field, generates a non-equilibrium plasma in gases at atmospheric or lower pressures. The plasma is generated between two ferroelectric plates, or one ferroelectric plate and a second one made of other material (metal or semiconductor). In this second case, there is strong sputtering and excitation of atoms from metallic electrode. It causes that such a plasma emits an useful light for spectroscopic measurements (line strengths, branching ratios, etc.) $[2,3]$. The source is in non-equilibrium state, and most of its power goes to light emission, not in heating of the gas.

\section{Experimental measurements and results}

\subsection{Experimental setup}

The "ferroelectric" plasma was generated between two plates of ferroelectric ceramics, separated by approximately half a millimeter gap. The working gas flew between them from one end to another. The input was by the end opposite to the monochromator. Alternating voltage $(5 \mathrm{kV}, 50 \mathrm{~Hz})$ was applied to the electrodes. 
Because of periodic nature of the "ferroelectric" plasma, the signal measured from plasma source was time averaged over the short time near the maximum of the light intensity. Light was collected by two lenses, imaged onto input slit $(0.15 \mathrm{~mm})$ of the $1 \mathrm{~m} \mathrm{McPherson}$ monochromator. The monochromator was equipped with the standard $1200 \mathrm{G} / \mathrm{mm}$ grating blazed for $300 \mathrm{~nm}$ and with an UV sensitive photomultiplier placed behind the $100 \mu \mathrm{m}$ wide output slit. The signal from the photomultiplier was averaged by the Princeton Applied Research Boxcar Averager. The output boxcar signal was sampled and stored on a 4-channel digital oscilloscope.

Two working-gas mixtures were used: air, and mixture of air with few percent (about $4 \%$ ) of argon. A qualitative analysis of time integrated emission spectra shows some intense molecular bands in the region from 200 to $400 \mathrm{~nm}$ (a maximum around $350 \mathrm{~nm}$ ). The $\mathrm{N}_{2}^{+}, \mathrm{N}_{2}, \mathrm{NO}$ and $\mathrm{OH}$ bands were detected, the last one due to ambient humidity of the air which was used as plasma-forming gas. The spectra were collected during an adequately slow continuous monochromator scanning $(1 \mathrm{~nm} / \mathrm{min})$. In order to trigger the boxcar by the center of the the plasma period, the light reflected from the input slit blades was collected by a quartz fiber and detected by a second photomultiplier. The output signal from this photomultiplier was used as a trigger. Bands of the second positive system of nitrogen molecule $C^{3} \Pi_{\mathrm{u}}-B^{3} \Pi_{\mathrm{g}}$ in $\Delta v=-2,-1$, and 0 sequences were studied in both gas mixtures. The monochromator spectral band-pass $(0.1$ to $0.2 \mathrm{~nm}$ ) was low enough to recover partially resolved structure of the emission band for determination of both vibrational and rotational temperatures. The monochromator apparatus function was measured by means of a low-pressure Hg lamp.

\subsection{Analysis of results}

Spectra of the second positive system were analyzed by means of computer programs searching for the best $T_{\mathrm{v}}$ and $T_{\mathrm{r}}$ temperatures to fit correctly a theoretically calculated spectrum to the experimental band shape.

Two different fitting procedures were used: the first one (marked as " $A$ ") has been elaborated in the Institute of Plasma Physics, Czech Academy of Sciences, and the second one (" $B$ ") originates from the University of Orleans. No correction of the band intensity distribution for the spectral sensitivity of the optical system has been made. However, in our case, the spectral sensitivity for spectra analysis can be considered as a constant because of the proper combination of the photomultiplier spectral sensitivity and the grating blaze efficiency (the spectral sensitivity was checked by a continuous spectrum tungsten strip lamp).

Using the " $A$ " procedure $[4,5]$ the rotational temperatures were found by fitting the beginning of $\Delta v=-2,-1$ or 0 sequences after numerical smoothing and continuum subtraction. The numerical model has been based on the high-resolution analysis of the rotational system [6,7] and on the $H_{n, l}$ London factors [8]. The $(0,2),(0,1)$ and $(0,0) \mathrm{N}_{2}$ bands were used for the $T_{\mathrm{r}}$ determination.

For vibrational temperatures, the technique consisted in a generation of band-profiles for given both $T_{\mathbf{r}}$ and instrumental function, and subsequent subtraction from experimental spectra. The process was repeated till the difference between simulated and experimental spectra was smaller than required. Resulting 
relative populations of the $C^{3} \Pi_{\mathrm{u}}$ state in two vibronic levels of $v=0$ and $v=1$ determined the $T_{v}$ value.

Using the " $B$ " procedure [9-11], the rotational and vibrational temperatures have been found by unique common fit over a large complex spectrum of the overlapping $\mathrm{N}_{2}$ bands covering $\Delta v=-2,-1$ and 0 sequences, without any preliminary numerical smoothing, any baseline subtraction and supposing the apparatus function as unknown. The numerical band simulation was based on the same literature data as in procedure " $A$ ". The computer program is capable to simulate the spectrum of the $C^{3} \Pi_{\mathrm{u}}-B^{3} \Pi_{\mathrm{g}}$ transition without limit of wave numbers, $v^{\prime}$ and $v^{\prime \prime}$, but in order to gain a time, we have simulated only the overlapped $(0,0),(1,1)$, $(2,2),(3,3),(4,4),(1,0),(2,1)$, and $(3,2)$ bands between the collected signal wave numbers. In order to determine the $T_{\mathrm{r}}$ and $T_{\mathrm{v}}$ temperatures from a given real (and noisy) spectrum, the best simulated spectrum was compared point-by-point using a best-fit criterion with four free parameters: $T_{\mathrm{r}}, T_{\mathrm{v}}$, apparatus width (supposed Gaussian) and background level. To test a noise sensitivity of the temperature estimator, we simulated some noisy spectra with the help of random numbers for the apparatus profile corresponding to the Gaussian width of DX $=7 \mathrm{~cm}^{-1}$ (half-width at 1/e height), the noise-to-signal ratio of $0.025,0.05$ and 0.1 and the $T_{\mathrm{r}}=T_{\mathrm{v}}=3 \mathrm{kK}$. The best-fit criterion is found to be the good one, since the relative standard deviation of the temperatures are at the same order of magnitude as the noise-to-signal ratio: $\Delta T / T=0.015,0.025$ or 0.04 , respectively.

\subsection{Results}

Figure 1 presents two measured and corresponding two simulated spectra (procedure " $B$ ") from two working gases. The vibrational and rotational temperatures found in both cases are given in Table. The experimental results concerning

\section{TABLE}

Experimental characteristics of the "ferroelectric" plasma in two different gas mixtures.

\begin{tabular}{c|c|c}
\hline \hline Zone & $T_{\mathrm{v}}$ & $T_{\mathrm{r}}$ \\
\hline Air & & \\
\hline Model $A$ & $2800 \pm 200 \mathrm{~K}$ & $450 \pm 100 \mathrm{~K}$ \\
Model $B$ & $2800 \pm 100 \mathrm{~K}$ & $650 \pm 100 \mathrm{~K}$ \\
\hline Air+argon & & \\
\hline Model $A$ & $3200 \pm 300 \mathrm{~K}$ & $900 \pm 100 \mathrm{~K}$ \\
Model $B$ & $3300 \pm 200 \mathrm{~K}$ & $800 \pm 100 \mathrm{~K}$
\end{tabular}

the first fitting procedure are marked as " $A$ ", those concerning the second procedure are marked as " $B$ ". One can see from the table that in air rotational and vibrational temperatures are lower than in the case of mixture (air with $\mathrm{Ar}$ ), which can be explained by increasing of the power in plasma because argon lowers the resistance of the plasma. 

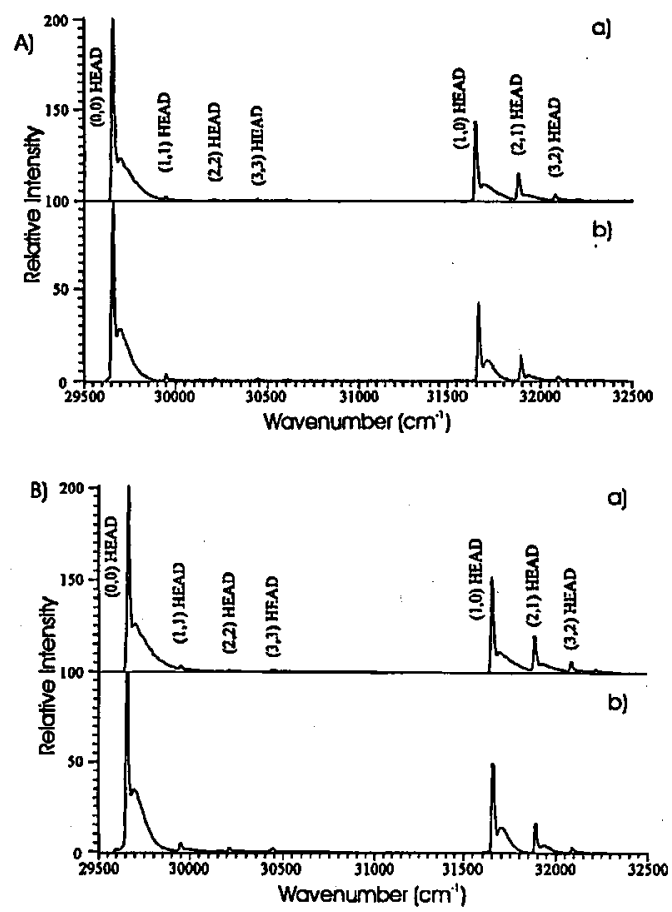

Fig. 1. Comparison of the simulated and observed spectra for both gas mixtures: (A) air, (B) air + Ar, (a) simulated spectra, (b) observed spectra.

\section{Conclusions}

In our experimental conditions, non-equilibrium nature of the "ferroelectric" plasma is evident. Differences in rotational and vibrational temperatures show a similarity of this discharge to a microwave discharge [12], even if the last one is produced in quite different pressures and frequencies. The difference of the temperatures, which shows the departure of the plasma state from the local thermal equilibrium (LTE) or partial LTE, may be however quite easily influenced by changing the gas pressure, applied voltage or frequency.

This plasma may be very useful as a spectroscopic excitation source with low Doppler widths and quite high excitation energy.

\section{References}

[1] J. Kusz, in: Advances in Low-Temperature Plasma Chemistry, Technology and Applications, Vol. 4, Ed. H.V. Boenig, Technomic, Lancaster, PA 1992, p. 237.

[2] A. Goly, J. Kusz, B. Nguyen Quang, S. Weniger, J. Quant. Spectrosc. Radiat. Transf. 45, 157 (1991).

[3] J. Kusz, Astron. Astroph. Suppl. Ser. 92, 517 (1992).

[4] M. Simek, Acta Phys. Slov. 42, 83 (1992). 
[5] S. DeBenedictis, G. Dilecce, M. Simek, Acta Phys. Univ. Comeniae 33, 159 (1992).

[6] F. Roux, F. Michaud, M. Vervloet, J. Mol. Spectrosc. 67, 143 (1989).

[7] F. Roux, F. Michaud, M. Vervloet, J. Mol. Spectrosc. 158, 270 (1993).

[8] I. Kovacs, Astrophys. J. 145, 643 (1966).

[9] A. Czernichowski, J. Phys. D 20, 559 (1987).

[10] H. Nassar, A. Czernichowski, J. Phys. (France) 51, C5-289 (1990).

[11] H. Nassar, A. Czernichowski, Acta Phys. Pol. A 84, 215 (1993).

[12] V.M. Donnelly, D.L. Flamm, G. Collins, J. Vac. Sci. Technol. 21, 817 (1982). 